\title{
ÜBER DAS CARNOSIN UND SEINE METHYLVERBINDUNGEN IN DEN MUSKELN VON GIFTSGHLANGEN TRIMERESURUS FLAVOVIRIDIS FLAVOVIRIDIS ("HABU") SOWIE ÜBER IHRE PHARMAKOLOGISCHEN WIRKUNGEN*
}

\author{
SHIGERU TSUNOO, KAZUYOSHI HORISAKA, YUKIO MIYASHITA, \\ JUNIGHI TAKEDA UND NAOHIKO TAKATAMA \\ Pharmakologisches Institut der Medizinischen Fakultät der Showa Universität, Shinagawa-ku, Takya
}

Eingegangenen am 13. April 1964

Seit langem nimmt man im allgemeinen an, dass Carnosin ( $\beta$-Alanylhistidin) und seine Methylverbindungen, Anserin ( $\beta$-Alanyl-1-N-methylhistidin) und Ophidin ( $\beta$-Alanyl-2C-methylhistidin), nur in den Muskelextrakten der Wirbeltiere als charakteristische Substanzen enthalten sind (1-3). Die interessanten Ergebnisse über die Verteilung dieser Dipeptide und ihren Gehalt (4-6) in Muskeln der verschiedenen Tiere wurden schon gewonnen, während vieles ihrer physiologischen und pharmakologischen Wirkungen (7-10) jedoch noch unbekannt bleibt. Es ist vor allem sehr interessant, dass Ophidin, welches bis jetzt nur aus den Schlangenmuskeln (3, 11-13) abgetrennt wurde, auch aus anderen Geweben ausser Muskeln isoliert wurde. Neulich trennten Tsunoo und andere weisse, nadelförmige Kristalle aus gefrorenem Walpankreas (14) und Walmuskel (15) ab, welche aus der Elementaranalyse nach den Salzbildungen, Spaltungsversuchen durch $\mathrm{HBr}$ und IR-Spektrum von ihren Pikrolonaten als Ophidin identifiziert wurden. Aus den oben erwähnten Tatsachen seien eingehende Untersuchungen über Verteilung dieser Dipeptide und ihren Gehalt in Muskel und in anderen Geweben der verschiedenen Tierarten ein wertvoller Helfer, um ihre physiologischen und pharmakologischen Wirkungen zur Klarheit zu kommen.

In vorliegenden versuchen haben wir das Vorkommen dieser Dipeptide in der Muskulatur der Giftschlange "Habu" und ihre pharmakologischen Wirkungen untersucht.

\section{VERSUCHSTEILE}

\section{Die Isolierung und Identifizierung des Carnosins und des Ophidins} aus der Muskulatur dor Giftschlange "Habu"

Matcrial: 17 Stück "Habu" (12 kg), an Okinawa Insel gefangen, wurden zur Untersuchung herangezogen. Die Rumpfteile von "Habu" wurden nach der Entfernung der Haut und Eingeweiden einige Tage lang in $80 \%$ igem Alkohol eingetaucht. Dann 
wurden die Rumpfteile so lange mit heissem Wasser behandelt, bis die Ablösung der Muskeln von den Skeletten möglich war. Dit Gewichtsdifferenz vor und nach der Wasseirextraktion entsprach der Menge der verwendeten Muskeln. Sodann wurden 3 $\mathrm{kg}$ Muskeln von "Habu" in der Tat gebraucht.

Fraktionicrung der freien Aminosäuren: Man brachte den Wasscrextrakt mit Alkoholextrakt zusammen. Der vereinigte Extrakt wurde nach dem in Tab. 1 gezeigten Verfahren fraktioniert. Nach der Enteiweissung durch Tannin-Blei-Methode wurde das Filtrat vom Blei beseitigt und durch eine Säule mit Dowex $50\left(\mathrm{H}^{+}\right)$geschickt, um alle Aminosäuren und Peptide zu adsorbieren. Anschliessend wurde die Säule mit Wasser gespült. Die adsorbierten Aminosäuren wurden $2 \mathrm{~N} \mathrm{NH}_{3}$-Lösung eluiert. Das Eluat wurde im Vak. unter der Hinfügung des Wassers mehrmals eingeengt, um überschüssiges $\mathrm{NH}_{3}$ zu entfernen. Diesc wässrige Lösung wurde mit Amberlite IRC-50, zu pH 7.0 und 4.8 kontrolliert, säulenchromatographisch behandelt. Dadurch wurde die Histidin-Frak-

Tabrila 1. Fraktionierungsverfahren der Extrakte von Schlangenmuskeln.

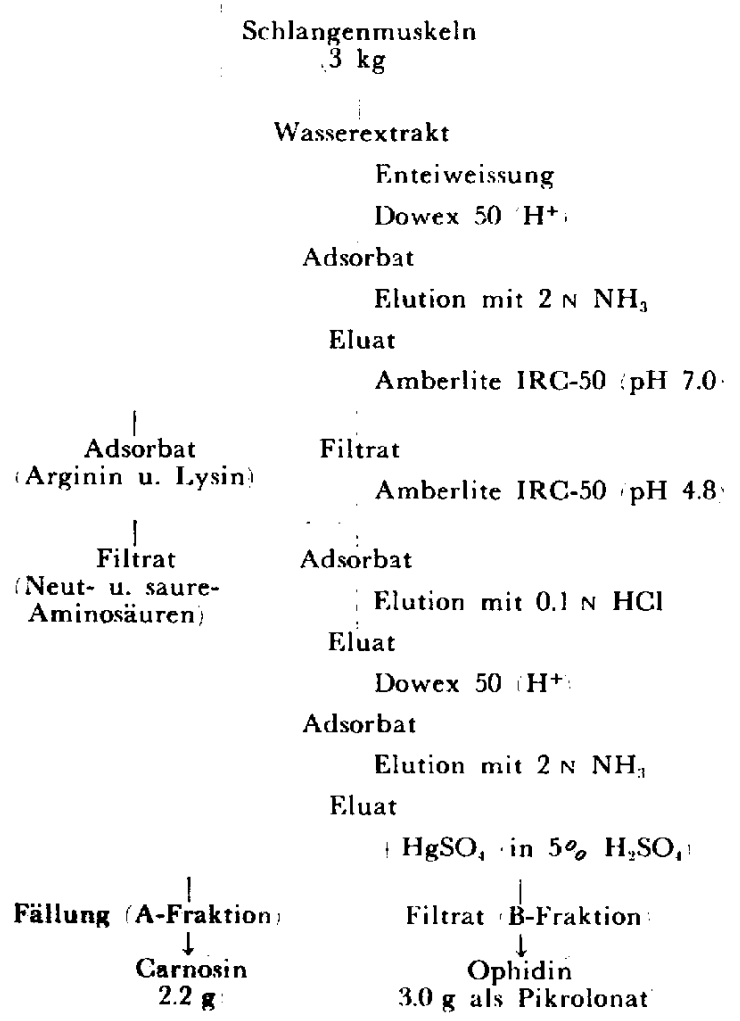

tion als Adsorbat von Lysin- und Arginin-Fraktion sowic derselben der neutralen und sauren Aminosä ıren getrennt. Diese Histidin-Fraktion wurde durch 0.1 N HCl-Lösung eluiert. Das Eluat wurde mit Dowex $50\left(\mathrm{H}^{+}\right)$behandelt. Die adsorbierten Stoffe wurden nach Wasserspülung durch $2 \mathrm{~N} \mathrm{NH}-$ Lösung cluicrt. Man engte das Eluat im Vak. cin, bis freie Kristalle sich ausschieden. 
Lösungsmittcl für Prpicrchromatographie: n-Butanol: Essigsäure : Wasser (Abkürzung, Bu/Ac) $80: 20: 20$, Pyridin: Wasser (Py) 65:35, Pyridin: n-Butanol: Wasser (Py/Bu) $80:$ $20: 20$, Phenol (Phen), gesättigt mit einer wässrigen Lösung von $6.3 \%$ igem Na-citrat und $3.7 \circ$ oigem $\mathrm{KH}_{2} \mathrm{PO}_{4}$.

Papier. Toyo Nr. 51.

Nachweis von Dipeptiden durch 0.2 o.oige Ninhydrinlösung in Butanol. Carnosin durch Paulysche Diazoreaktion.

In dieser Histidin-Fraktion wurden zwei Ninhydrin-positive Substanzen papierchromatographisch betrachtet. Für die Trennung dieser Substanzen verwendeten wir HgSO, (in $5 \%$ iger $\mathrm{H}_{2} \mathrm{SO}_{4}$ ).

A-Fraktion ( $\mathrm{HgSO}_{4}$-Fällung): Nach der Zerlegung durch $\mathrm{H}_{2} \mathrm{~S}$ wurde das Schwefelquecksilber abfiltriert. Das Filtrat wurde nach der Entfernung des Schwefelwasserstoffs durch Dowex $50\left(\mathrm{H}^{+}\right)$behandelt. Anschliessend wurde die Säule mit Wasser gespült, das Adsorbat mit $2 \mathrm{~N} \mathrm{NH}_{3}$-Lösung eluiert. Diese Eluat-Fraktion wurde im Vak. eingeeengt. Man bekam weisse, nadelförmige Kristalle, die einen Schmelzpunkt von 252 (unter Zers.) besitzen. Ausbeute $2.2 \mathrm{~g}$.

Die Analysen ergaben folgendes:

$$
\begin{array}{rrrr}
\mathrm{C}_{9} \mathrm{H}_{14} \mathrm{O}_{3} \mathrm{~N}_{4} & & & \\
\text { Ber. } & \text { C } 47.78 & \mathrm{H} 6.24 & \mathrm{~N} 24.77 \\
\text { Gef. } & 47.90 & 6.57 & 24.41
\end{array}
$$

Diese Substanz zeigt eine Paulysche Diazoreaktion. Zieht man nun die erwähnten Tatsachen und die folgende Papierchromatographie in Betracht, so wird diese Substanz

TABELLLE 2. Rf-Wert.

\begin{tabular}{lcccc}
\hline & Bu/Ac & Py & Py/Bu & Phen \\
Isolierte Substanz & 0.13 & 0.52 & 0.09 & 0.78 \\
Carnosin & 0.13 & 0.52 & 0.08 & 0.78 \\
\hline
\end{tabular}
für Carnosin angesehen (Tab. 2).

B-Fraktion (HgSO,-Filtrat): Das Filtrat wurde mit $\mathrm{H}_{2} \mathrm{~S}$ zerlegt. Die von Schwefelquecksilber abfiltrierte Lösung wurde durch Dowex $50\left(\mathrm{H}^{+}\right)$behandelt. Das

Adsorkat wurde nach der Wasserspülung mit $2 \mathrm{~N} \mathrm{NH}_{3}$-Lösung eluiert. Das Eluat wurde im Vak. unter der Hinfügung des Wassers mehrmals eingeengt, um überschüssiges $\mathrm{NH}_{3}$ zu entfernen. Diese basische wässrige Lösung wurde mit alkoholischer Pikrolonsäurelösung bis zu neutraler Reaktion versetzt. Dabei schieden sich $3.0 \mathrm{~g}$ gelbe, tafelförmige Kristalle, die bei 208" (unter Zers.) schmolzen und zeigten folgende Analysenwerte.

$$
\begin{aligned}
& \mathrm{C}_{10} \mathrm{H}_{16} \mathrm{O}_{3} \mathrm{~N}_{4} \cdot \mathrm{C}_{10} \mathrm{H}_{8} \mathrm{O}_{5} \mathrm{~N}_{4} \cdot \mathrm{H}_{2} \mathrm{O} \\
& \text { Ber. C } 45.97 \text { H } 5.01 \quad \text { N } 21.45 \\
& \begin{array}{llll}
\text { Gef. } & 45.98 & 5.19 & 21.52
\end{array}
\end{aligned}
$$

Paulysche Diazoreaktion dieser freien Substanz ist negativ. Sie besitzt einen Schmelzpunkt von $245 C^{\circ}$ (unter Zers.) und folgende Analysenwerte.

$$
\begin{aligned}
& \mathrm{C}_{10} \mathrm{H}_{10} \mathrm{O}_{3} \mathrm{~N}_{4} \cdot \mathrm{H}_{2} \mathrm{O} \\
& \text { Ber. } \quad \text { C } 46.50 \text { H } 7.02 \quad \text { N } 21.69 \\
& \begin{array}{llll}
\text { Gef. } & 46.54 & 6.93 & 22.07
\end{array} \\
& 46.31 \quad 7.15
\end{aligned}
$$


T.ABELI.F. 3. Rf-Wert.

\begin{tabular}{lcccc}
\hline & Bu/Ac & Py & Py/Bu & Phen \\
Isolierte Substanz & 0.14 & 0.65 & 0.10 & 0.93 \\
Ophidin & 0.14 & 0.65 & 0.10 & 0.93 \\
\hline
\end{tabular}

Auch bei der Ergebnissen der papierchromatographischen Untersuchungen erwies sich sie als identisch mit Ophidin (Tab. 3).

\section{Einflüsse des Carnosins, Anserins und Ophidins auf die Herztätigkcit, den Blutdruck und die Atmung}

Material und Methodik: Die zu prüfenden Dipeptide, Carnosin, Anserin und Ophidin, wurden aus der Natur dargestellt und isoliert. Die chemischen Konstitutionen dieser Substanzen sind aus der folgenden Tabelle 4 ersichtlich.

Tabri.J.e 4. Carnosin und seine Derivate.

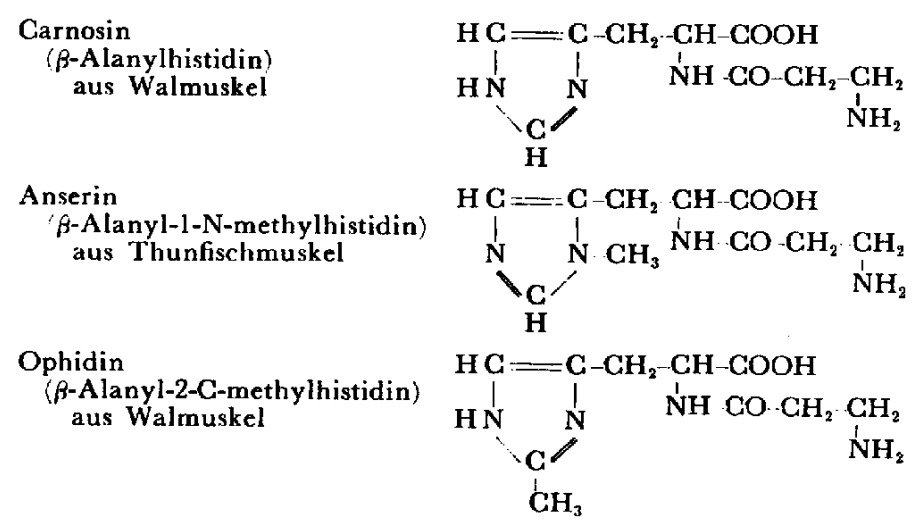

Wir verwendeten für die Versuche über die Einfüsse der oben angegebenen Substanzen auf den Kreislauf und die Atmung Kaninchen im Gewicht von ungefähr $2 \mathrm{~kg}$. Kaninchen wurden mit 1.0-1.2 g Urethan/kg s.c. narkotisiert, dann in Rückenlage gebracht. Die Atmung wurde mit einer Marey-Kapsel, der Blutdruck mit einem $\mathrm{Hg}$ Manometer sowie Karotiskatheter, und der Herzschlag in situ durch direkte Aufhängung der Herzspitze unter Benutzung des Fadens gleichzeitig registriert. Die Dipeptide, in $0.1 \mathrm{ml}$ von physiologischer Kochsalzlösung gelöst, wurden intravenös injiziert. Die Registrierung der Bewegung des isolierten Kaninchenherzens wurde durch LangendorffGunnsche Methode ermittclt. Als die Durchströmungsflüssigkcit wurde dic mit Sauerstoff gesättigte Locke-Ringersche Lösung gebracht. Dic Dipeptide, in der verschiedenen Konzentrationen in $0.1 \mathrm{ml}$ Wasser gelöst, wurden in den Gummischlauch, welcher an die Herzkanüle angeschlossen wurde, zur Vermeidung von der 1)ruckveränderung der crnährenden Flüssigkeit sehr langsam injiziert.

Experimente in situ (Abb. IA.B.C.. 2A.B.C.): Der Blutdruck wurde durch intravenöse Injektion dieser drei Dipeptide unter $5 \mathrm{~ms} / \mathrm{kg}$ nicht verändert. Erst in 1 osen von 20 my/kg machte sich eine leichte Steigerune des Blutdrucks bemerkhar. welche nach 3 


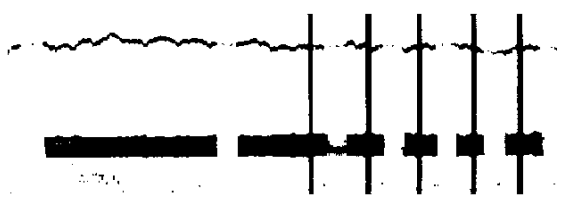

$\uparrow$ Carnosin $5 \mathrm{mg} / \mathrm{kg}$ Kaninchen $20 \mathrm{~kg}$ 站

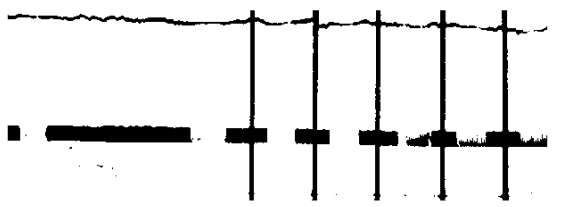

$\uparrow$ Carnosin $20 \mathrm{mg} / \mathrm{kg}$ Kaninchen $1.9 \mathrm{~kg} \quad \hat{\sigma}$

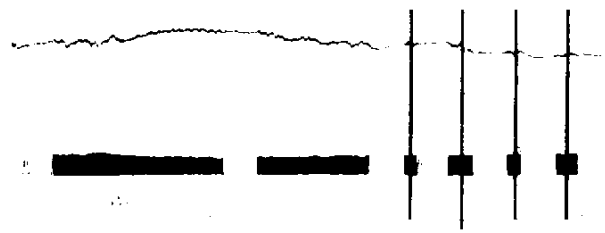

$\uparrow$ Carnosin $40 \mathrm{mg} / \mathrm{kg}$ Kaninchen $2.2 \mathrm{~kg}$ \& ABв. IA. Blutdruck und Atmung.

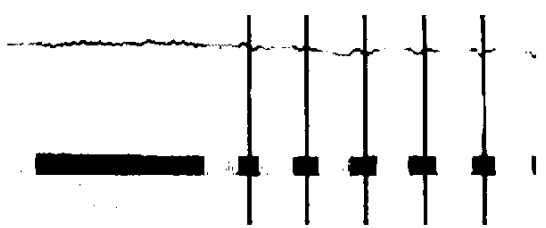

$\uparrow$ Anserin $5 \mathrm{mg} / \mathrm{kg}$ Kaninchen $2.2 \mathrm{~kg} \delta$

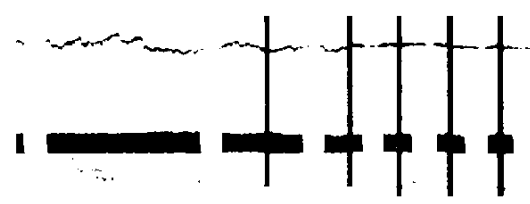

$\uparrow$ Anserin $20 \mathrm{mg} / \mathrm{kg}$ Kaninchen $1.9 \mathrm{~kg} \hat{\sigma}$

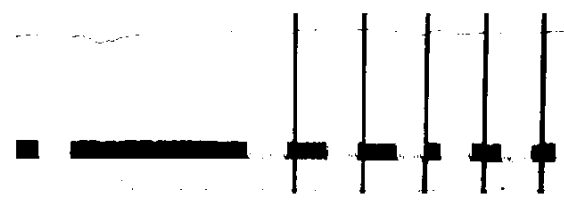

$\uparrow$ Anserin $40 \mathrm{mg} / \mathrm{kg}$ Kaninchen $2.1 \mathrm{~kg} \delta$ ABB. 1B. Blutdruck und Atmung.

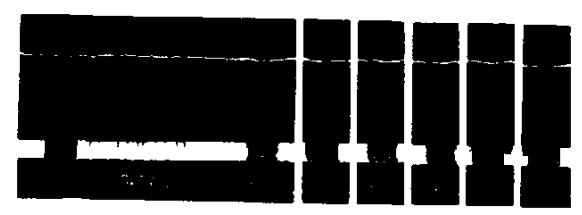

$\uparrow$ Ophidin $5 \mathrm{mg} / \mathbf{k g}$ Kaninchen $1.9 \mathrm{~kg} \hat{o}$

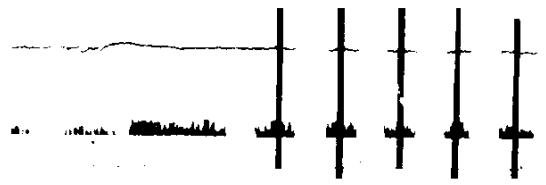

$\uparrow$ Ophidin $20 \mathrm{mg} / \mathrm{kg}$ Kaninchen $1.9 \mathrm{~kg}$ f

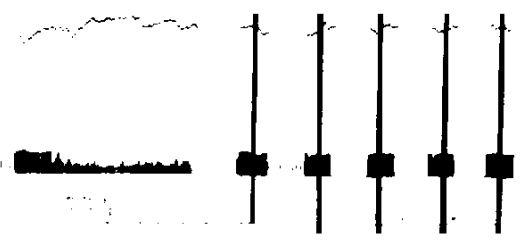

$\uparrow$ Ophidin $40 \mathrm{mg} / \mathrm{kg}$ Kaninchen $1.9 \mathrm{~kg}$ 令

ABв. 1C. Blutdruck und Atmung.

Minuten wieder auf die Norm gebracht wurde. In Dosen von $40 \mathrm{mg} / \mathrm{kg}$ trat eine anhaltende Blutdrucksteigerung ein, deren Normalisierung ziemlich viele Zeit in Anspruch n.1hin. 

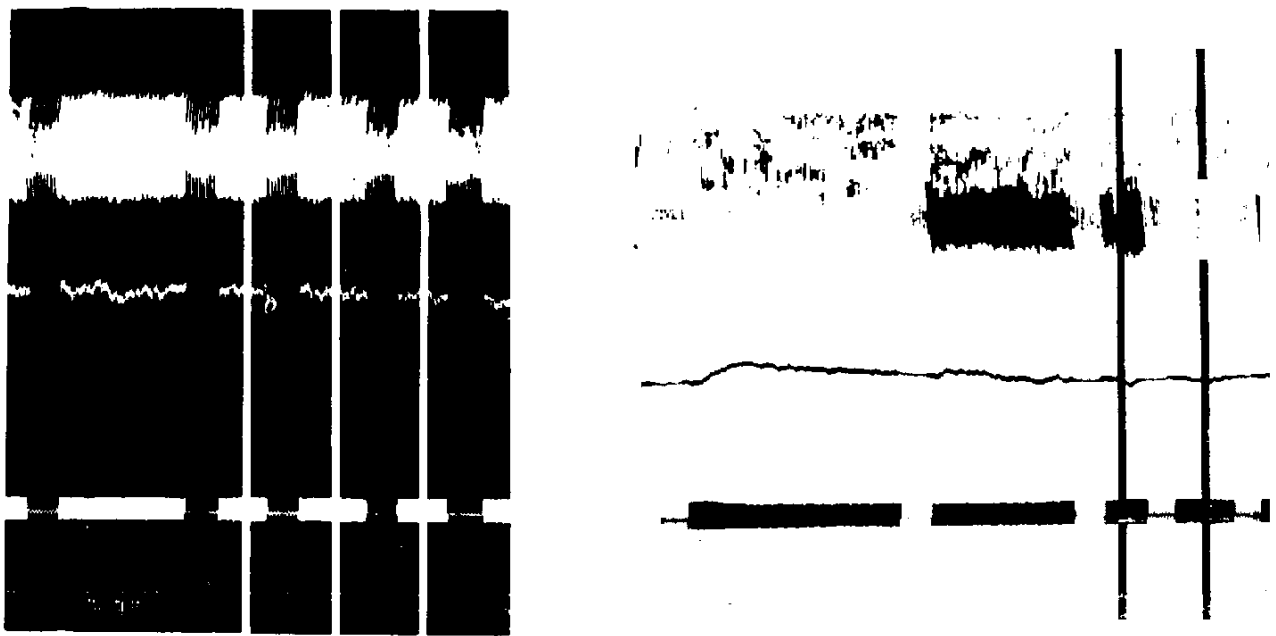

$\uparrow$ Carnosin $5 \mathrm{mg} / \mathrm{kg}$ Kaninchen $2.7 \mathrm{~kg}$ f $\uparrow$ Carnosin $20 \mathrm{mg} / \mathrm{kg}$ Kaninchen $2.7 \mathrm{~kg} \&$

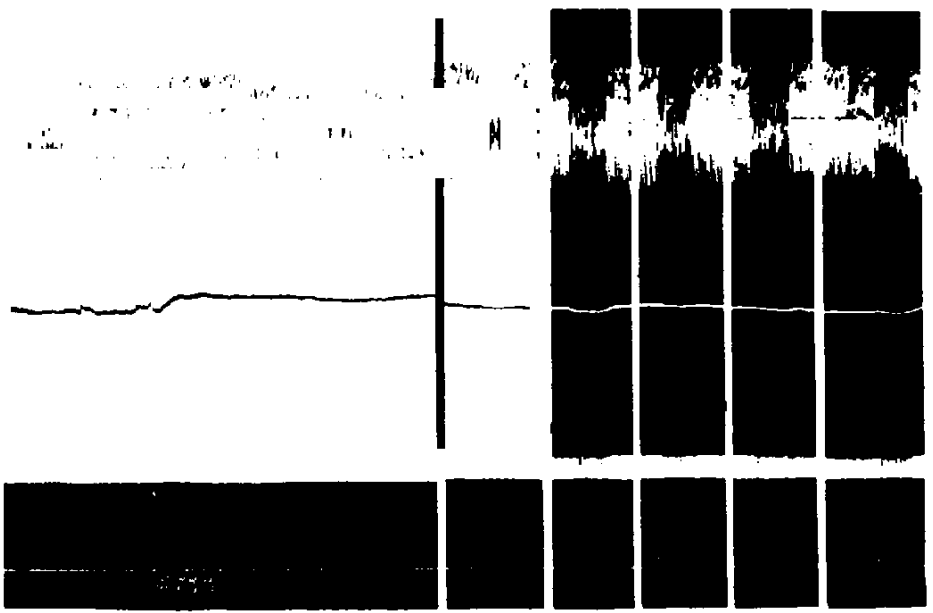

$\uparrow$ Carnosin $40 \mathrm{mg} / \mathrm{kg}$ Kaninchen $2.6 \mathrm{~kg}$ \&

ABB. 2A. Blutdruck, Atmung und Herzbewegung.

In allgemeinen riefen diese Substanzen keine wesentliche Änderung in der Atmung hervor. Aber in Dosen $40 \mathrm{mg} / \mathrm{kg}$ wurde eine leichte Hemmung der Atmung beobachtet.

Während die Schlagzahl des Herzens fast keine Beeinflussung hervorrief, wurde leichte Verkleinerung der Amplitude in Dosen von $40 \mathrm{mg} / \mathrm{kg}$ beobachtet.

Untersuchungen an isoliertem Kaninchenherzen (Abb. 3A.B.C.): Drei Dipeptide riefen in Dosen von 0.01-0.1 mg auf das isolierte Kaninchenherz leichte Hemmung hervor. In Dosen von $1 \mathrm{mg}$ wurde Hemmung nach vorübergehender Stimulierung beobachtet. Leichte Verengerung der Koronargefässe stellte sich von Dosen von $0.01 \mathrm{mg}$ ein, und zwar geht die Verengerungsgrad zur Vergrösserung der applizierten Mengen der Dipeptide fast in proportionaler Beziehung. 

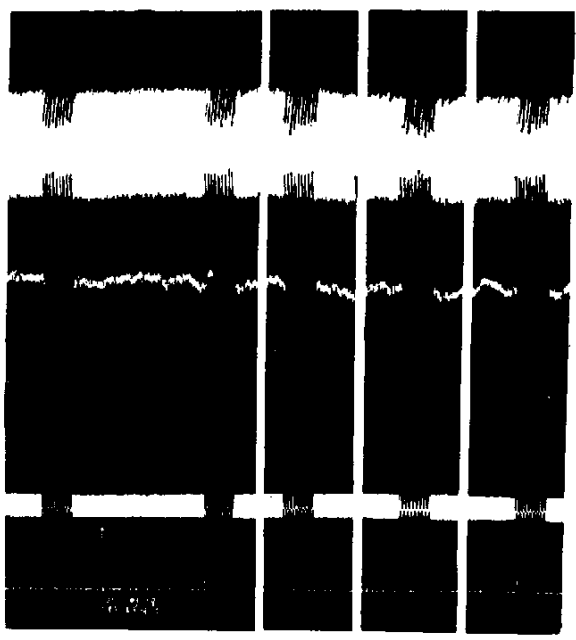

$\uparrow$ Anserin $5 \mathrm{mg} / \mathrm{kg}$ Kaninchen $2.7 \mathrm{~kg}$ \&

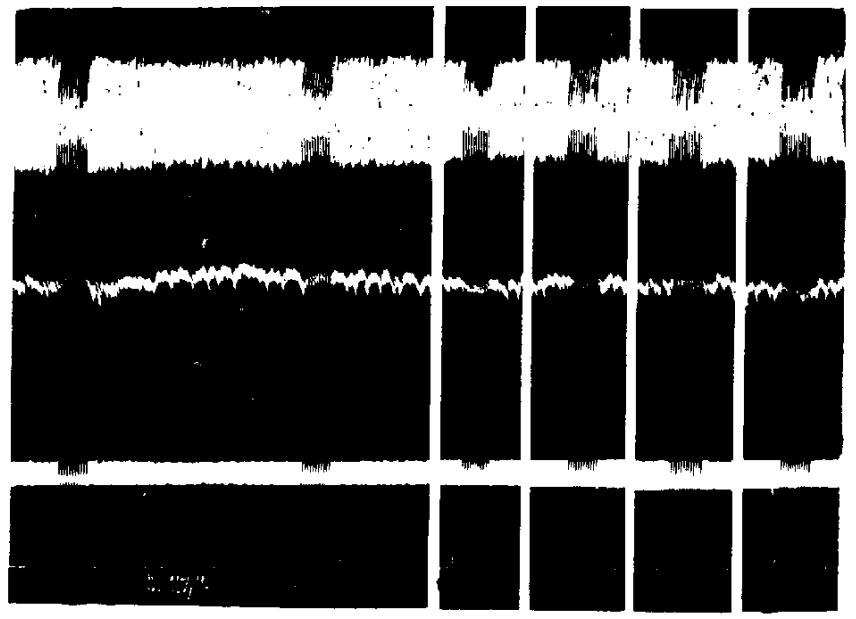

$\uparrow$ Anserin $20 \mathrm{mg} / \mathrm{kg}$ Kaninchen $2.6 \mathrm{~kg}$ \&

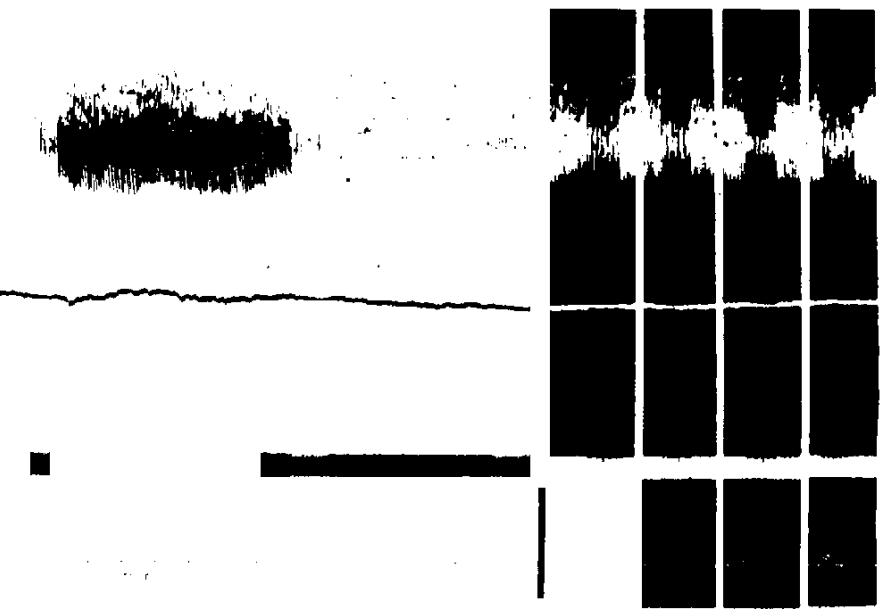

$\uparrow$ Anserin $40 \mathrm{mg} / \mathrm{kg}$ Kaninchen $2.6 \mathrm{~kg} \delta$

ABB. 2B. Blutdruck, Atmung und Herbbewegung. 


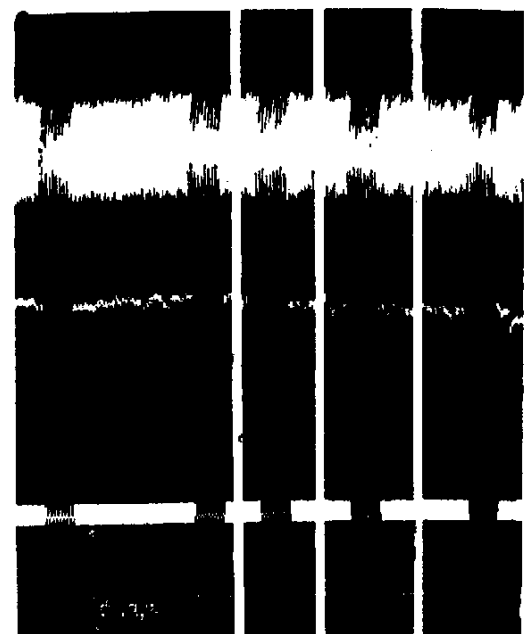

ABB. 2C. Blutdruck, Atmung und Herzbewegung.

$\uparrow$ Ophidin $5 \mathrm{mg} / \mathrm{kg}$ Kaninchen $2.7 \mathrm{~kg}$ 就

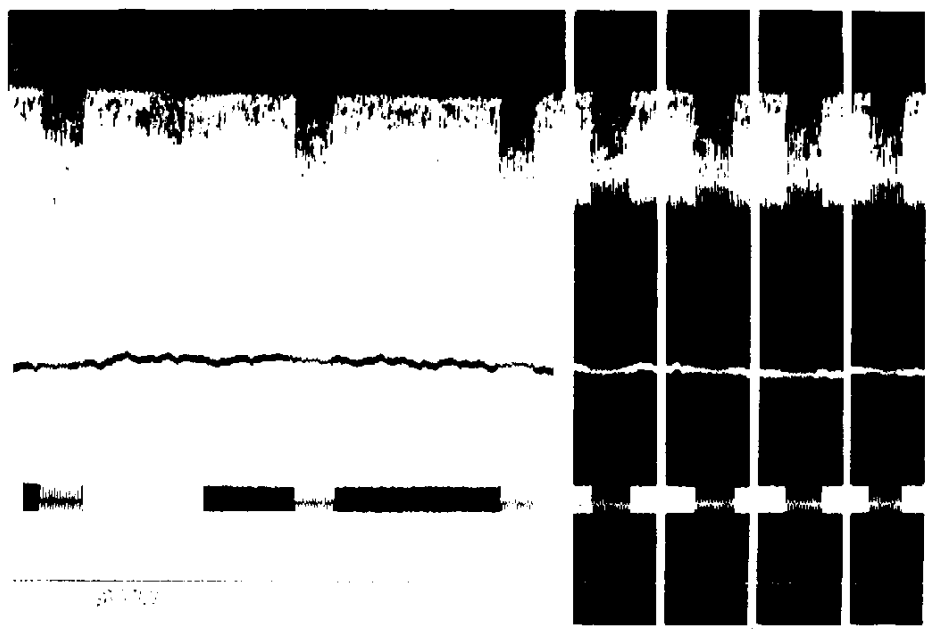

$\uparrow$ Ophidin $20 \mathrm{mg} / \mathrm{kg}$ Kaninchen $2.7 \mathrm{~kg}$ \&

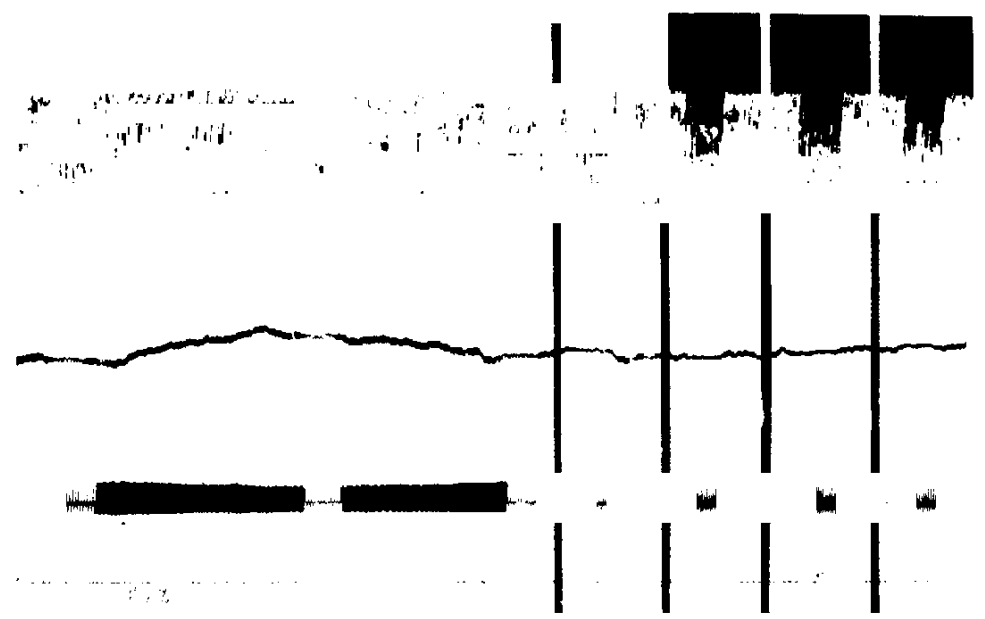

$\uparrow$ Ophidin $40 \mathrm{mg} / \mathrm{kg}$ Kaninchen $2.7 \mathrm{~kg} \delta$ 


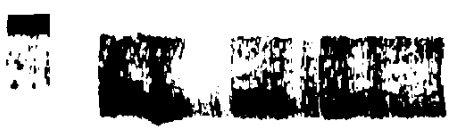

$\uparrow$ Carnosin $0.01 \mathrm{mg}$

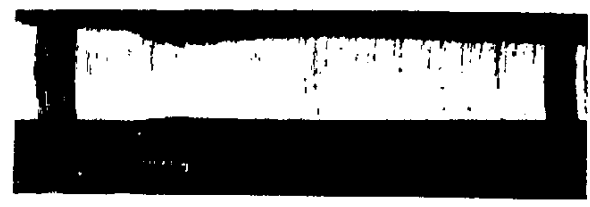

$\uparrow$ Carnosin $0.1 \mathrm{mg}$

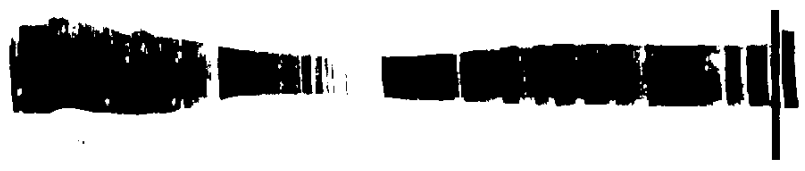

$\uparrow$ Carnosin $1 \mathrm{mg}$

ABE. 3A. Untersuchungen an isoliertem Kaninchenherzen.

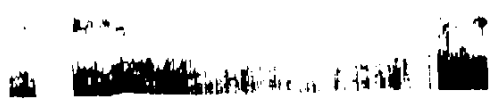

$\uparrow$ Anserin $0.1 \mathrm{mg}$

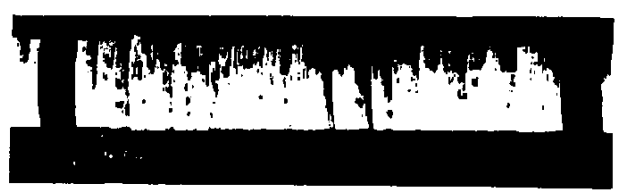

$\uparrow$ Anserin $0.1 \mathrm{mg}$

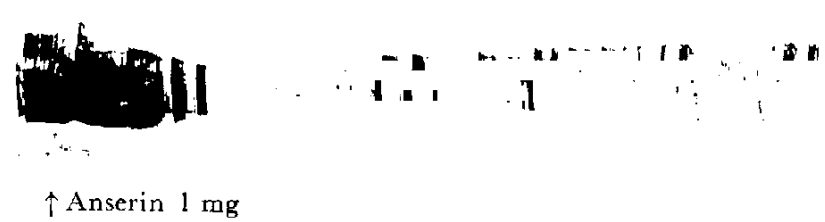

Aвв. 3B. Untersuchungen an isoliertem Kaninchenherzen. 


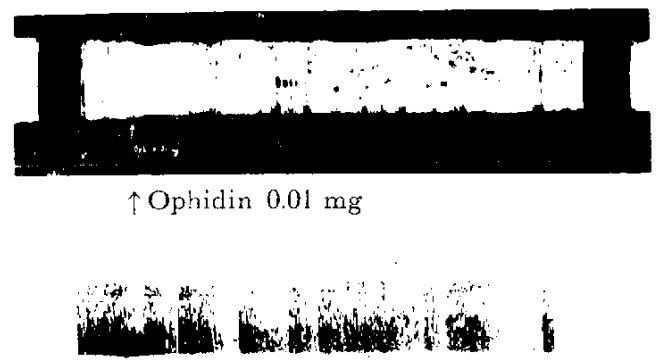

$\uparrow$ Ophidin $0.1 \mathrm{mg}$

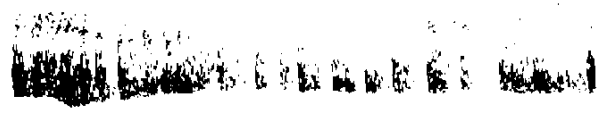

$\uparrow$ Ophidin $1 \mathrm{mg}$

ARB. 3C. Untersuchungen an isoliertem

Kaninchenherzen.

\section{DISKUSSION}

Seit das Ophidin aus den Muskeln der giftigen und ungiftigen Schlangen in Formosa (3) entdeckt wurde, fand sich es auch in den Muskeln von japanischen Giftschlange Agkistrodon Blomhoff (11) neben Histidin bekanntlich. Aus den Muskeln von Natrix tigrina tigrina (12) wurden Ophidin und Carnosin kristallinisch dargestellt, während Carnosin aus denselben von Seeschlangen Laticauda semifasciata und laticaudata (13) nicht isoliert wurde, sondern nur Histidin und 2-C-Methylhistidin wurden ausser Ophidin papierchromatographisch nachgewiesen. Dazu kam, dass Carnosin an dem Gehalt Ophidin überlegen war Ausser den oben genannten Schlangenmuskeln wurden Ophidin und 2-Methylhistidin aus Walpankreas $(14,16)$, und Carnosin wurde neben Ophidin aus Walmuskel (15) dargestellt.

Was die physiologischen und pharmakologischen Wirkungen dieser Dipeptide, Carnosin, Anserin und Ophidin, anbetrifft, so lässt sich zunächst an die Entfaltung ihrer Wirkungen in Muskeln mit der Begründung denken, dass sie in etwas grösserer Anreicherung in Muskeln anzutreffen sind (7-10). Durch das Vorkommen dieser Dipeptide im Hirn $(17,18)$ und Auge $(19,20)$ ist das Interesse für ihre Wirkungen in steigendem Masse in den Vordergrund gerückt. Vor kurzem wurde nach Ichihara und anderen (21) untersucht, wie diese Dipeptide die Muskelkontraktion beeinflussen. Diese Versuche wurden am isolierten Uterus und Eileiter des Huhns, der Ratte und des Mecrschweinchens vorgenommen. Es stellte sich heraus, dass sich ihre Wirkungen je nach Rasse der Tiere und bei verschiedenen Sorten dieser Dipeptide sehr unterschiedlich beeinflussen liessen. Im Jahre 1936 beobachteten du Vigneaud und anderer (22) eine Senkung des Blutdrucks nach der Applikation des -Carnosins an der Katze und dem Kaninchen, 
mit Amytal und Luminal narkotisiert. Weiter zeigten sie, dass beim dl-Carnosin die Blutdrucksenkung nur halb so stark war, und beim enantiomorphen $d$-Carnosin fehlte. Aber unsere geschilderten Versuche zeigten, dass die Blutdrucksenkung durch die Dipeptide, Carnosin, Anserin und Ophidin, am Kaninchen fehlte, vielmehr die Blutdrucksteigerung sich als alle gemeinsame Wirkung dieser Dipeptide entfalten liess.

\section{ZUSAMMENFASSUNG}

1. Aus den Muskeln von "Habu" wurden Ophidin ( $3.0 \mathrm{~g}$ als Pikrolonat) und Carnosin (2.2 g) kristallinisch dargestellt, und sie wurden durch Analyse und Papierchromatographie identifiziert.

2. Die Wirkungen des Carnosins und seiner Methylverbindungen, Anserins und Ophidins, auf die Herztätigkeit, den Blutdruck und die Atmung in situ sowie auf das isoliertes Herz wurden am Kaninchen untersucht. Kein Unterschied der Wirkungen bestand in drei Dipeptiden, indem sie die Blutdrucksteigerung als gemeinsame Wirkung zeigten.

Anerkennung : Die zu den Versuchen benötigten Giftschlangen "Habu" wurden von Herrn T. Ire in Okinawa und von den Studienreise nach Okinawa angetretenen Studenten, Herrn Y. Ban, H. Yoshida und $\mathbf{K}$. Inoue freundlicherweise zur Verfügung gestellt, wofür wir an dieser Stelle danken.

\section{LITERATUR}

1) Gulewitsch, W. und Amiradzibi, S. : Ber. disch. chem. Ges. 33, 1902 (1900)

2) Ackermann, D., Timpe, O. Und Poll.er, K. : Z. phys. Chem. 183, 1 (1929)

3) Imamurs, H.: J. Biochem., Tokyo 30, 479 (1939)

4) Wol.FF, W.A. UND WH.SON, W. : J. biol. Chem. 109, 565 (1935)

5) LukTon, A. UND Ol.cotT, H.S. : Food Res. 23, 611 (1958)

6) DAveY, C.L. : Arch. Biachem. Biophys. 89, 303 (1950)

7) BATE SMITH, E.C. : J. Physiol. 92, 336 (1938)

8) DAvey, C.L. : Arch. Biochem. Biophys. 89, 296 (1960)

9) QURESHI, Y. UND WOOD, T. : Biochem. biophys. acta 60, 190 (1962)

10) Brown, D.E.S., Carew, E.B. uni Bauer, R.S. : Aich. Biochem. Biophys. 100, 45 (1963)

11) Tомгт, T. : Z. phys. Chem. 304, 72 (1956)

12) Tsuneo, Sh. UnI, TAKeDA, J. : Folia pharmacol. japon. 58, 31\$ (1962)

13) Tsunoo, Sh., Horisaka, K., Motonishi, K. unid TAkeda, J. : J. Biochem., Takyo 56, 603 (1934)

14) Tsunox), Sil., Musasil, A. und Horisaka, K.: Proc. Japan Acad. 35, 485 (1959)

15) Horisaka, K. Uni) Musasili, A. : J. Biochem., Tokyo 53, 271 (1963)

16) Musasili, A. : Showa Med. J. 20, 887 (1960)

17) Hosinin, E.A. und Smart, M. : Canad. J. Bicchem. Physiol. 38, 569 (1969)

18) Tsunoc, Sil., Horisaka, K., Kawasumi, M., Aso, K. uni) Tokul, Sh. :J. Bixhem., Tokyo 54, 355 (1963)

19i Lont;, C. : Biochemists' Handknok S. 705, E \& F. N. Spon Ltd., London (1961)

20. Tsunko, Sil., Horisaka, K., Sasaki, Sil., Aso, K. Uni) Tokul, Sh. : J. Biochem., Tokya 54, 363(19.33)

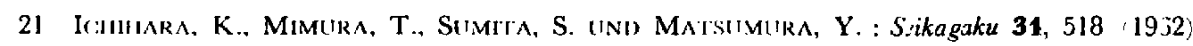

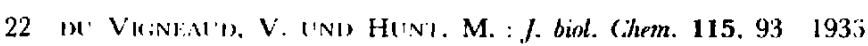

